\section{Preliminary findings on the effect of light-oxidation on Asiago d'allevo vecchio protected designation of origin cheese}

\section{Stefania Balzan, Luca Fasolato, Barbara Cardazzo, Filomena Montemurro, Enrico Novelli}

Dipartimento di Biomedicina Comparata e Alimentazione, Università degli Studi di Padova, Legnaro (PD), Italy

\section{Abstract}

Food is exposed to light during processing, packaging, distribution and retail storage, resulting in deterioration of the product quality. Milk and other dairy products are among the most sensitive due to the high content of riboflavin, vitamin $B_{2}$, which is an efficient photosensitizer for oxidative processes. Photooxidation in cheese induces degradation of nutritional quality such as proteins, lipids, and vitamins. Moreover, it causes formation of off-flavours, off-odours and gradual bleaching.

The objective of this study was to evaluate the effect of light exposition on sliced Asiago d'allevo cheese (lowland $v s$ alpine) aerobically packaged stored for $336 \mathrm{~h}$ under both fluorescent light and in the dark. Alpine cheese showed higher $b^{*}(\mathrm{P}<0.001)$ and $a^{*}$ indexes $(\mathrm{P}<0.001)$ and also a higher lipid oxidation $(\mathrm{P}<0.01)$ than lowland one. Riboflavin content was significantly higher $(\mathrm{P}<0.001)$ in lowland cheese. Cheese samples exposed to the light were significantly lighter $(\mathrm{P}<0.001)$ than those exposed in the dark and they also showed a decrease in yellowness $(\mathrm{P}<0.001)$ and redness $(\mathrm{P}<0.001)$ as well as in riboflavin content $(\mathrm{P}<0.001)$. Lipid oxidation did not produce statistically significant change. Storage time significantly affected riboflavin content $(\mathrm{P}<0.001)$, TBARs $(\mathrm{P}<0.001)$ and cheese colour $(\mathrm{P}<0.001)$. Results suggest that light exposition had a pronounced effect on cheese characteristics.

\section{Introduzione}

Negli ultimi dieci anni si è osservato un trend positivo nell'acquisto di formaggi che meglio di altri alimenti hanno risposto alle richieste dei consumatori in termini di freschezza, funzione d'uso, innovazione e prezzo. La quasi totalità degli acquisti viene effettuata presso la grande distribuzione organizzata e il consumatore è disposto a spendere per un maggior contenuto di servizio che, per i formaggi, è dato dalla porzionatura e dal grattugiato fresco presentati con varie modalità di confezionamento (ISMEA, 2011).

La luce costituisce una delle maggiori cause di scadimento nutrizionale e sensoriale in quanto è responsabile della degradazione di proteine, grassi e vitamine con sviluppo di odori sgradevoli (sunlight odour e sunlight flavour), perdita di colore e formazione di composti potenzialmente dannosi per la salute del consumatore (es. ossidi del colesterolo). L'esposizione dei prodotti lattiero-caseari alla radiazione luminosa avviene in tutte le fasi della loro vita commerciale, dalla produzione fino alla commercializzazione, e indipendentemente dalla presenza del packaging.

La fotossidazione può procedere attraverso due meccanismi, le reazioni di Tipo I e Tipo II, che possono svilupparsi anche in maniera competitiva. Le reazioni di Tipo I procedono secondo la formazione di radicali liberi e tendono a essere più frequenti a basse concentrazioni di ossigeno (He et al., 1998). In quelle di Tipo II l'energia è trasferita all'ossigeno tripletto dalle sostanze fotosensibilizzanti eccitate con formazione di ossigeno singoletto che è estremamente reattivo e attacca i composti organici senza la loro preliminare conversione in radicali (Foote, 1991). Generalmente, le reazioni di Tipo I sono catalizzate dalla presenza di raggi UV-lontano (300-200 nm) o da luce caratterizzata da alta energia (10.000-100.000 lx) come quella solare; sono quindi poco frequenti negli alimenti esposti in quanto le luci fluorescenti dei banchi espositori generano una limitata frazione di raggi UV che vengono inoltre assorbiti dal packaging (Mortensen et al., 2004). Nei prodotti lattiero-caseari sono naturalmente presenti sostanze fotosensibilizzanti come riboflavina (vitamina $\mathrm{B}_{2}$ ), porfirina e clorofilla che inducono l'inizio dell'ossidazione durante l'esposizione alla luce visibile (Intawiwat et al., 2011) favorendo le reazioni di Tipo II.

L'Asiago d'allevo denominazione d'origine protetta (D.0.P.) è un formaggio a pasta semicotta prodotto nei territori delle province di Vicenza e Trento e in alcune zone delle province di Treviso e Padova. A seconda della durata della stagionatura l'Asiago d'allevo può essere denominato mezzano ( $4 / 6$ mesi), vecchio (superiore a 10 mesi) e stravecchio (superiore a 15 mesi). La tradizionale produzione in alpeggio è stata mantenuta sino a oggi e il formaggio stagionato in stabilimenti ubicati al di sopra dei $600 \mathrm{~m}$ s.l.m. e ottenuto con latte derivante da stalle collocate al di sopra della stessa quota, può fregiarsi della menzione di Prodotto della montagna (www.asiagocheese.it). L'Asiago d'allevo è commercializzato al libero servizio sia preconfezionato in atmosfera protettiva o sottovuoto, sia preincartato con pellicola direttamente avvolta sulla porzione 0 con
Correspondence: Stefania Balzan, Dipartimento di Biomedicina Comparata e Alimentazione, Università degli Studi di Padova, piazzetta del donatore 4, 35020 Legnaro (PD), Italy.

Tel: +39.049 .8272966 - Fax: +39.049 .8272604 .

E-mail: stefania.balzan@unipd.it

Key words: Photooxidation, Asiago d'allevo PD0 cheese, Riboflavin, TBARs, Color.

Conflict of interests: the authors declare no potential conflict of interests.

Acknowledgments: the authors thank Andrea Benetti and Greta Pretto for their helpful support.

Received for publication: 15 January 2013.

Revision received: 8 July 2013.

Accepted for publication: 9 July 2013.

This work is licensed under a Creative Commons Attribution 3.0 License (by-nc 3.0).

(C) Copyright S. Balzan et al., 2013

Licensee PAGEPress, Italy

Italian Journal of Food Safety 2013; 2:e40

doi:10.4081/ijfs.2013.e40

vassoio e pellicola in presenza di uno spazio di testa di dimensioni variabili.

Scopo del lavoro è stato valutare la suscettibilità alla fotossidazione del formaggio Asiago d'allevo preincartato mantenuto in situazione di commercio. Tale modalità di confezionamento è infatti frequentemente adottata presso i punti vendita in virtù del notevole consumo di tale prodotto.

\section{Materiali e Metodi}

La valutazione ha preso in considerazione $n$. 2 forme di Asiago d'allevo di 12 mesi prodotte in caseifici ubicati rispettivamente in pianura (provincia di Vicenza) e in montagna (Altopiano dei Sette Comuni, VC). La forma di montagna è stata ottenuta a partire da latte di bovine in alpeggio alimentate con foraggio fresco e una minima integrazione in concentrati mentre quella di pianura deriva da latte di bovine alimentate con unifeed senza impiego di insilati.

Da ciascuna forma, dopo l'apertura in laboratorio, è stato ottenuto un parallelepipedo privato della crosta e di $1 \mathrm{~cm}$ di sottocrosta da cui sono state ricavate mattonelle delle dimensioni di $13,0 \times 7,0 \times 0,5 \mathrm{~cm}$, del peso di circa $45 \mathrm{~g}$ ciascuna. Si è quindi proceduto a creare dei vassoi per simulare il preincartato successivamente esposti alla luce o mantenuti al buio, in regime di refrigerazione. Al fine di ridurre la variabilità dovuta al posizionamento dei vassoi soprattutto in presenza di illuminazione, in 
ogni vassoio sono state inserite una fetta di formaggio di pianura e una di formaggio di montagna e quindi ciascun vassoio è stato avvolto con pellicola in PVC (KOEX 412) idonea per alimenti.

Immediatamente dopo il confezionamento, tutti i vassoi sono rimasti al buio in cella frigo $\left(4 \pm 1^{\circ} \mathrm{C}\right)$ per 62 ore prima dell'esposizione alla luce. Successivamente n.14 vassoi sono stati mantenuti ancora al buio in cella frigo a $4 \pm 1^{\circ} \mathrm{C}$ e umidità relativa del $74,5 \%$ circa e n. 15 vassoi sono stati collocati in un frigo espositore (a $3{ }^{\circ} \mathrm{C} \pm 1$ e umidità relativa dell' $85 \%$ circa) allo scopo di simulare le condizioni di vendita. L'illuminazione è stata effettuata con luce neon Master TL-D Super 80 G13 36W (temperatura di colore: $6500^{\circ} \mathrm{K}$; indice di resa cromatica: $85 \mathrm{Ra}$ ) dalle $6: 30$ a.m. alle 8:30 p.m.

All'apertura delle forme sono stati determinati composizione centesimale, contenuto di sale (AOAC, 2000) e profilo acidico della frazione lipidica (Novelli et al., 2002). I parametri indicativi dei fenomeni ossidativi sono stati valutati dopo il preliminare stoccaggio al buio di tutti i campioni. I campionamenti sono stati effettuati dopo 0, 6, 24, 48, 72, 166, 216, $336 \mathrm{~h}$ di esposizione alla luce prelevando i vassoi secondo un ordine che riducesse eventuali effetti dovuti alla diversa distanza dalla fonte luminosa. Ciascun campionamento ha preso in considerazione due confezioni esposte alla luce e due conservate al buio.

Il colore è stato misurato dopo esposizione all'aria $\left(1 \mathrm{~h}, 2 \pm 2^{\circ} \mathrm{C}\right)$ su 5 diverse zone della fetta, con uno spettrocolorimetro (C508; Minolta Co., Osaka, Japan) settato sull'illuminante D65 (luce del giorno standard) osservatore a $10^{\circ} \mathrm{C}$. I dati sono stati espressi secondo il sistema colorimetrico CIELAB $L^{*} a^{*} b^{*}$ (Kristensen et al., 2001; www.cie.co.at/cie/). La determinazione dei prodotti secondari dell'ossidazione (TBARs) è stata effettuata in doppio su ciascun campione secondo il metodo di Chozas et al. (1993). La misura del contenuto in riboflavina è stata condotta in triplicato secondo il metodo proposto da Havemose et al. (2004).

I dati sono stati sottoposti ad analisi della varianza (ANOVA) utilizzando il pacchetto statistico PASW Statistics 18 (2009). La distribuzione dei dati è stata valutata con il test di Kolmogorov-Smirnov. Il test post hoc è stato effettuato con il Tukey test la cui differenza tra le medie è significativa al livello 0,05 . L'analisi ha considerato l'effetto dell'origine, dell'esposizione, del tempo e le relative interazioni sui fenomeni ossidativi a carico del prodotto.

\section{Risultati}

Il formaggio di pianura si caratterizza per un maggiore contenuto in sostanza secca, proteine, grasso e per una differente composizione in acidi grassi insaturi, in particolare coniugati dell'acido linoleico e C18:1 $t 11$ loro principale precursore, che risultano essere tendenzialmente in minor quantità (Tabella 1). Gli effetti principali dell'origine del prodotto (Tabella 2) e del tempo di esposizione (Tabella 3) hanno comportato differenze sempre statisticamente significative; la modalità di esposizione (buio vs luce, Tabella 2) ha determinato cambiamenti significativi $(\mathrm{P}<0,001)$ solo a carico del colore strumentale

Tabella 1. Composizione centesimale (\% sostanza secca) e contenuto in acidi grassi (\% sul totale degli identificati) del formaggio Asiago d'allevo vecchio ( $n=2$ forme).

Pianura Montagna

\begin{tabular}{lcc} 
Composizione centesimale & & \\
$\quad$ Sostanza secca & 73,9 & 70,4 \\
Proteina grezza & 41,4 & 44,2 \\
Grasso & 48,1 & 45,9 \\
Ceneri & 6,7 & 6,5 \\
Sale & 2,8 & 2,6 \\
Acidi grassi & & \\
SFA & 55,6 & 52,0 \\
MUFA & 26,2 & 28,7 \\
PUFA & 4,3 & 5,4 \\
ECLA & 0,6 & 1,2 \\
Cl8:1 nl1t & 1,9 & 2,9 \\
\hline
\end{tabular}

SFA, $\Sigma$ acidi grassi saturi; MUFA, $\Sigma$ acidi grassi monoinsaturi; PUFA, $\Sigma$ acidi grassi polinsaturi; $\varepsilon$ CLA, sommatoria coniugati acido linoleico.

Tabella 2. Effetto dell'origine $(n=29)$ e della modalità di esposizione $(n=29)$ su colore strumentale $\left(\right.$ CIELAB $\left.L^{*} a^{*} b^{*}\right)$, contenuto di riboflavina (unità fluorescenza/g di formaggio) e prodotti secondari dell'ossidazione lipidica.

\begin{tabular}{|c|c|c|c|c|c|c|c|c|}
\hline & & 0 & & & & ESM & & \\
\hline & Pianura & & Montagna & Buio & Luce & & 0 & E \\
\hline$L^{*}$ & 71,2 & & 67,7 & 68,8 & 70,1 & 0,2 & $* * *$ & $* * *$ \\
\hline$a^{*}$ & 0,5 & & 1,1 & 0,87 & 0,70 & 0,15 & $* * *$ & $* * *$ \\
\hline$b^{*}$ & 15,2 & & 18,7 & 17,7 & 16,2 & 0,03 & $* * *$ & $* * *$ \\
\hline TBARs $\mathrm{A}_{450 \mathrm{~nm}}$ & 0,21 & & 0,26 & 0,25 & 0,23 & 0,2 & $*$ & ns \\
\hline TBARs $A_{532 \mathrm{~nm}}$ & 0,12 & $\forall$ & 0,16 & 0,14 & 0,14 & 0,1 & $* *$ & ns \\
\hline Riboflavina & $2,9 \times 10^{5}$ & $1,8 \times 105$ & $2,5 \times 10^{5}$ & $2,2 \times 10^{5}$ & $7 \times 10^{3}$ & $* * *$ & $* *$ & \\
\hline
\end{tabular}

0 , origine; E, esposizione; ESM, errore standard medio; TBARs, prodotti secondari dell'ossidazione; $\mathrm{A}_{450}$, assorbanza ordine zero a 450; $\mathrm{A}_{532 n m}$, assorbanza ordine zero a 532 nm; ns, non significativo. *P<0,05; ** $<<0,01$; $* * * \mathrm{P}<0,001$.

Tabella 3. Effetto del tempo di esposizione su colore strumentale (CIELAB L*a*b*; $\mathbf{n}=29$ ), prodotti secondari dell'ossidazione lipidica e contenuto di riboflavina.

\begin{tabular}{|c|c|c|c|c|c|c|c|c|c|c|}
\hline & \multicolumn{10}{|c|}{ Tempo di esposivione (h) $(\mathrm{n}=29)$} \\
\hline & 0 & 6 & 24 & 48 & 72 & 168 & 216 & 336 & ESM & $\mathbf{P}$ \\
\hline $\mathrm{L}^{*}$ & $71,0 \mathrm{a}$ & $69,4 \mathrm{~b}$ & $71,5 \mathrm{a}$ & $71,4 \mathrm{a}$ & $68,9 \mathrm{~b}$ & $69,0 \mathrm{~b}$ & $68,7 \mathrm{~b}$ & $65,7 \mathrm{c}$ & 0,3 & $* * *$ \\
\hline$a^{*}$ & $0,1 \mathrm{c}$ & $0,8 \mathrm{bc}$ & $0,9 \mathrm{bc}$ & $1,1 \mathrm{a}$ & $1,0 \mathrm{ab}$ & $0,9 \mathrm{bc}$ & $0,7 \mathrm{~b}$ & $0,8 \mathrm{bc}$ & 0,1 & $* * *$ \\
\hline $\mathrm{b}^{*}$ & $19,7 \mathrm{a}$ & $16,7 \mathrm{c}$ & $18,0 \mathrm{~b}$ & $17,1 \mathrm{c}$ & $17,1 \mathrm{c}$ & $16,4 \mathrm{~cd}$ & $15,5 \mathrm{~d}$ & $15,0 \mathrm{e}$ & 0,2 & $* * *$ \\
\hline TBAA $_{450}$ & $0,345 \mathrm{ab}$ & $0,394 \mathrm{bc}$ & $0,243 \mathrm{abc}$ & $0,224 a b c$ & $0,136 \mathrm{c}$ & $0,120 \mathrm{c}$ & $0,214 a b c$ & 0,233abc & 0,03 & $* * *$ \\
\hline TBAA $_{532}$ & $0,238 \mathrm{ab}$ & $0,259 \mathrm{a}$ & $0,145 b$ & $0,063 \mathrm{bc}$ & $0,001 \mathrm{c}$ & $0,070 \mathrm{bc}$ & $0,145 b$ & $0,169 \mathrm{bc}$ & 0,02 & $* * *$ \\
\hline $\begin{array}{l}\text { Riboflavina } \\
\text { (unità fluorescenza/g } \\
\text { formaggio) }\end{array}$ & $2,9 \times 10^{5} \mathrm{a}$ & $3,0 \times 10^{5} \mathrm{a}$ & $2,6 \times 10^{5} \mathrm{ab}$ & $2,1 \times 10^{5} b$ & $2,6 \times 10^{5} \mathrm{ab}$ & $2,2 \times 10^{5} \mathrm{~b}$ & $1,9 \times 10^{5} \mathrm{bc}$ & $1,6 \times 10^{5} \mathrm{C}$ & $1,4 \times 10^{4}$ & $* * *$ \\
\hline
\end{tabular}

ESM, errore standard medio; TBA, acido tiobarbiturico; $\mathrm{A}_{450}$, assorbanza ordine zero a $450 ; \mathrm{A}_{522}$, assorbanza ordine zero a $532 ;$ ns, non significativo. ${ }^{*} \mathrm{P}<0,05 ; * * \mathrm{P}<0,01 ; * * * \mathrm{P}<0,001$. 
e del contenuto di riboflavina. Non si osservano differenze per quanto riguarda i prodotti secondari dell'ossidazione lipidica.

Il formaggio di montagna si presenta meno brillante e con indici del rosso e del giallo maggiori. I formaggi esposti alla luce mostrano un maggior valore di $L^{*}$ accompagnato da un minor valore di $a^{*}$ e $b^{*}$. La durata della conservazione comporta invece una progressiva riduzione di tutti gli indici e al passare del tempo si osserva un progressivo discoloramento dei formaggi. Diversamente dagli altri parametri, i prodotti secondari dell'ossidazione (espressi come Assorbanza ordine zero) a entrambe le lunghezze d'onda evidenziano un andamento altalenante con sensibile diminuzione dei prodotti secondari a $72 \mathrm{~h}\left(\mathrm{~A}_{532 \mathrm{~nm}}\right)$ di conservazione e successiva ricomparsa.

Il colore strumentale ha mostrato interazioni statisticamente significativa per $L^{*}$ (origine $\mathrm{x}$ tempo, $\mathrm{P}<0,001$; origine $\mathrm{x}$ tempo $\mathrm{x}$ esposizione, $\mathrm{P}<0,01$ ), $a^{*}$ (origine $\mathrm{x}$ esposizione, $\mathrm{P}<0,01$; origine $\mathrm{x}$ tempo, $\mathrm{P}<0,001$; esposizione $\mathrm{x}$ tempo, $\mathrm{P}<0,01$; origine $\mathrm{x}$ tempo $\mathrm{x}$ esposizione, $\mathrm{P}<0,05)$ e $b^{*}$ (origine $\mathrm{x}$ esposizione, $\mathrm{P}<0,01$; origine $\mathrm{x}$ tempo e origine $\mathrm{x}$ tempo $\mathrm{x}$ esposizione, $\mathrm{P}<0,001$ ).

In merito al contenuto di riboflavina, sono risultate significative le interazioni origine $\mathbf{x}$ tempo $(\mathrm{P}<0,001)$ e origine $\mathrm{x}$ esposizione $(\mathrm{P}<0,05)$ che evidenziano sempre un maggiore effetto sul formaggio di montagna. Benché l'interazione origine $\mathrm{x}$ esposizione $\mathrm{x}$ tempo non sia risultata statisticamente significativa, in Figura 1 appare evidente che il prodotto di montagna risente particolarmente, oltre che della luce, anche del passare del tempo di esposizione come appare della progressiva diminuzione della intensità di fluorescenza. Per l'analisi del TBARs a entrambe le lunghezze d'onda le interazioni non sono risultate statisticamente significative.

\section{Discussione}

L'indagine ha preso in considerazione formaggi a 12 mesi di stagionatura in quanto rappresentano una tipologia commercialmente piuttosto diffusa. Le differenze compositive riscontrate tra i due prodotti sono ascrivibili alla diversità della materia prima impiegata e alla tecnologia produttiva. Seppur entrambe le produzioni siano avvenute nel pieno rispetto dei requisiti stabiliti dal disciplinare di produzione dell'Asiago D.O.P è inevitabile che la modalità di scrematura del latte, le condizioni climatiche dei locali di stagionatura, la microflora lattica ed altri fattori siano diversi tra $\mathrm{i}$ caseifici (Segato et al., 2007).

Il diverso profilo lipidico è ascrivibile alla composizione acidica del latte, conseguente al rilevante introito di foraggio verde delle bovine

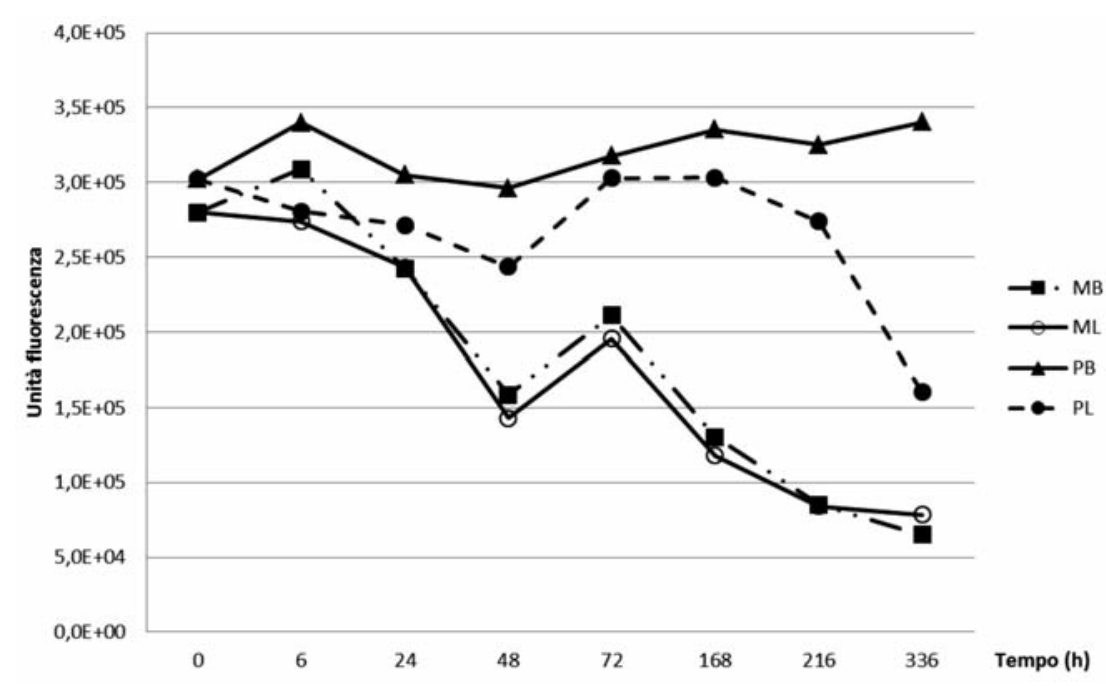

Figura 1. Variazione del contenuto di riboflavina (unità fluorescenza/g formaggio) durante l'esposizione. $\mathrm{MB}=$ formaggio di montagna conservato al buio; $\mathrm{ML}=$ formaggio di montagna esposto alla luce; $\mathrm{PB}=$ formaggio di pianura conservato al buio; $\mathrm{PL}=$ formaggio di pianura esposto alla luce.

mantenute al pascolo. Il consumo di elevate quantità di foraggio fresco comporta l'incremento della frazione insatura come conseguenza dell'elevato contenuto di acidi grassi polinsaturi (in prevalenza linoleico e linolenico) nell'erba (Bianchi et al., 2002; Balzan et al., 2007). Altri studi segnalano aumenti delle proporzioni degli acidi grassi polinsaturi nel latte di montagna collegati ad una ridotta attività di idrogenazione ruminale a carico degli acidi grassi dell'erba (Bugaud et al., 2001).

Come riportato in Introduzione, i formaggi interi o porzionati sono esposti alla luce dalla produzione alla commercializzazione e le radiazioni luminose caratterizzate da elevata energia (basse nelle regioni VIS/UV) sono in grado di determinare il maggior danno in quanto possono essere assorbite da numerose molecole presenti negli alimenti. Tali radiazioni non sono però tipiche delle lampade fluorescenti utilizzate nel settore alimentare e la scarsa quantità di raggi UV emessa dalle lampade dei banchi espositori viene generalmente assorbita dall'eventuale packaging (Mortensen et al., 2004). È però necessario ricordare che spesso il prodotto è confezionato con packaging trasparente al fine di permettere al consumatore di apprezzarne il contenuto.

Nel presente studio è stata impiegata una luce bianca fredda (prevalenti nello spettro di emissione comprese tra 400 e $500 \mathrm{~nm}$, blu e viola), solitamente usata per negozi ed uffici, che rappresenta il tipo di fonte luminosa più dannosa nei confronti degli alimenti. Si è scelto di porre il prodotto nella condizione di esposizione più critica in quanto la sostituzione delle lampade, talvolta, avviene senza molta cura da parte dell'operatore. Il confezionamento scelto ha invece simulato una delle modalità di preincarto presenti in commercio, ovvero mediante un vassoio ricoperto da pellicola PVC che lasciava una spazio di testa di circa $2 \mathrm{~cm}$ tra la superficie del formaggio e la pellicola garantendo anche una certa permeabilità all'ossigeno, indicativamente pari a 6000-9000 $\mathrm{cm}^{3} / \mathrm{m}^{2} / 24 \mathrm{~h}$ bar (Rocculi et al., 2003).

In merito all'effetto della luce e dell'atmosfera di confezionamento, i dati reperibili in letteratura riguardano prodotti diversi dall'Asiago d'allevo e interessano soprattutto formaggi molli, yogurt o burro. Inoltre, spesso non è neppure possibile confrontare le diverse condizioni di esposizione. La modesta differenza di temperatura tra l'esposizione al buio e quella alla luce non ha contribuito al diverso risultato tra le due modalità di conservazione in quanto, se evidente, un effetto della temperatura lo si potrebbe osservare solo a valori prossimi ai $30^{\circ} \mathrm{C}$ in virtù del limitato effetto della temperatura sulle reazioni fotossidative (Mortensen $e t$ al., 2004). Analogamente l'inevitabile differenza di umidità relativa dei due frigoriferi non ha inciso sugli aspetti ossidativi.

Il formaggio di montagna appare meno luminoso e più giallo rispetto a quello di pianura soprattutto per il diverso contenuto in carotenoidi presenti nell'alimento ingerito dalle bovine, in particolare nel foraggio fresco. Tale osservazione è stata confermata da vari autori (Segato et al., 2007; Jones et al., 2005). Tuttavia, i dati presenti in letteratura riguardanti il colore del formaggio appaiono scarsi e per gli stessi la luminosità non assume la medesima importanza che ha nelle carni. Sono infatti le componenti rosse e gialle a caratterizzare il prodotto (Jones et al., 2005).

I campioni mantenuti al buio sono meno luminosi ma con indici del rosso e del giallo 
più elevati. Tale andamento è stato rinvenuto anche in altri prodotti lattiero-caseari ed è probabilmente riconducibile a vari fattori. La componente rossa sembra aumentare per azione della fenolossidasi che comporta la formazione di composti melaninici (Mortensen et al., 2002) mentre la perdita della componente gialla nei formaggi esposti alla luce è dovuta all'azione di questa sui carotenoidi che agiscono da filtro interno e sulla riboflavina (Juric et al., 2003). Sono infatti carotenoidi e riboflavina a determinare il colore del formaggio.

La progressiva perdita di colore durante la conservazione, tipica dei formaggi porzionati, avviene per degradazione della riboflavina e dei carotenoidi ad opera della luce ma, nel caso del packaging impiegato, anche per la presenza di ossigeno dovuto alla metodica di confezionamento e alle caratteristiche della pellicola. Vari autori hanno riportato che pellicole poco permeabili all'ossigeno o conservazione in assenza di ossigeno mantengono costante il valore di $b^{*}$ (Juric et al., 2003; Kristensen et al., 2000).

La progressione dell'ossidazione lipidica è stata valutata attraverso la determinazione del TBARs, il cui comportamento non è facilmente confrontabile con quanto presente in letteratura a causa della peculiarità del prodotto. Come riportato in letteratura, nei formaggi la formazione del pigmento giallo $\left(\mathrm{A}_{450} \mathrm{~nm}\right)$ tende a prevalere su quella del pigmento rosa $\left(\mathrm{A}_{532}\right.$ $\mathrm{nm}$ ) a causa della presenza di aldeidi sature che non generano pigmenti rosa nella reazione con il TBA (Kristensen et al., 2001).

Il valore di assorbanza più elevato rispetto a quanto riportato da altri autori potrebbe essere dovuto anche alla formazione di composti come l'idrossimetilfurfurale che contribuiscono alla formazione di pigmento giallo mentre l'andamento irregolare del contenuto di TBARs, potrebbe indicare un continuo turnover di degradazione a carico della matrice lipidica (Mortensen et al., 2002). La maggior presenza di prodotti secondari dell'ossidazione nel formaggio di montagna è probabilmente dovuta anche al contenuto più elevato di acidi grassi insaturi di quest'ultimo. Mele et al. (2011) hanno infatti evidenziato un maggior livello di malonaldeide in formaggio Pecorino ricco in acidi grassi polinsaturi.

A differenza di altri lavori (Trobetas et al., 2008), nonostante la luce sia uno dei fattori predisponenti l'ossidazione lipidica, l'esposizione non ha comportato un sensibile incremento dei prodotti secondari dell'ossidazione $(P>0,10)$. Questi risultati sono confermati anche da Kristensen et al. (2000, 2001) e Kristensen et al. () che non hanno rilevato l'incremento dei livelli di TBARs in formaggio fuso cremoso neanche dopo 15 mesi di esposizione alla luce. Appare invece evidente l'effetto del tempo, che interessa sia le confezioni mantenute al buio sia quelle conservate alla luce, probabilmente anche a causa della presenza di ossigeno all'interno delle confezioni. In uno studio condotto su formaggio Graviera, Trobetas et al. (2008) hanno riportato un aumento dell'ossidazione lipidica in presenza di ossigeno. La totale esclusione dell'ossigeno dalle confezioni è però controversa in quanto atmosfere con il $100 \%$ di $\mathrm{CO}_{2}$ hanno comunque determinato irrancidimento e scadimento organolettico (Juric et al., 2003).

A differenza della lipolisi che comporta lo sviluppo di composti aromatici generalmente gradevoli e comunque tipici dei vari formaggi, l'ossidazione lipidica determina la comparsa di flavour tipicamente rancidi tra cui aroma oleoso, di sego e di cartone (Trobetas et al., 2008) oltre a determinare la formazione di composti dannosi per la salute del consumatore. È noto infatti che i prodotti di ossidazione del colesterolo e dei lipidi in generale sono considerati cancerogeni, mutageni e aterogenici (Mele $e t$ al., 2011). Benché i formaggi siano considerati meno predisposti all'ossidazione rispetto ad altri alimenti, per il profilo lipidico e per la presenza di antiossidanti naturali, questo fenomeno rappresenta comunque un aspetto critico che deve essere preso in considerazione durante la fase di conservazione e vendita del prodotto porzionato.

Le sostanze fotosensibilizzanti come riboflavina, clorofilla e porfirina assorbono facilmente la luce grazie alla presenza di doppi legami coniugati all'interno della propria struttura. Le reazioni fotossidative di Tipo II oltre alla presenza di fotosensibilizzatori, richiedono ossigeno alla base della formazione di ossigeno singoletto che reagisce con i grassi insaturi determinandone l'ossidazione.

L'iniziazione luce-dipendete comporta la degradazione della riboflavina presente generalmente nei formaggi in concentrazione variabile tra 0,30-0,60 mg/100 g (Møller e Saxholt, 1996).

Le intensità della luce degli armadietti di esposizione del formaggio nelle vendite al dettaglio degli Stati Uniti sono state determinate essere nella gamma di 1100-1900 lx (Deger e Ashoor, 1987). Generalmente le lampade presenti nei comuni banchi frigo dei supermercati presentano un'intensità di $1000 \mathrm{~lx}$. Il diverso livello di fluorescenza nei due formaggi può essere spiegato dalla diversa modalità di alimentazione della lattifere.

La degradazione della riboflavina, nel presente studio, si osserva dopo $24 \mathrm{~h}$ di esposizione. L'andamento non è prevedibile. Nel formaggio Havarti confezionato in atmosfera protettiva il decadimento si è osservato dopo 11 giorni di esposizione (Kristensen et al., 2000) mentre in una valutazione condotta sul formaggio fuso, il primo decadimento è avvenuto dopo 14 giorni per arrivare a una quota residua pari al 25\% della concentrazione iniziale dopo un anno di esposizione (Kristensen et al., 2001).

I dati aggregati mostrano in generale un maggior contenuto di riboflavina nel formaggio di pianura, la cui presenza sembra essere meno penalizzata dalla conservazione 0 dalla modalità di esposizione. Il maggior decadimento nei formaggi di montagna potrebbe essere dovuto alla presenza di una maggiore quantità di clorofilla e altre molecole in grado di trasformare l'ossigeno dallo stato di base al singoletto, con formazione di radicali liberi. La riboflavina è degradata a fino a circa $500 \mathrm{~nm}$. Il -carotene presente nel prodotto è in grado di fungere da filtro interno e proteggere la riboflavina dalla degradazione in particolare alle di 405 e $436 \mathrm{~nm}$ grazie alla sua azione di quenching sull'ossigeno tripletto-eccittato della riboflavina. Ciò comporta però un decadimento del -carotene (Cardoso et al., 2007). A differenza della confezione trasparente che offre una scarsa protezione, l'utilizzo di un packaging adeguato, ad esempio con fogli d'alluminio 0 in grado di assorbire la radiazione luminosa, potrebbe ridurre l'azione fotossidante (Intawiwat et al., 2011). Anche l'uso di packaging colorato (in ordine di efficacia decrescente: nero>marrone $>$ blu $>$ ross $0>$ giallo $>$ incolore) potrebbe limitare il danno (Mortensen et al., 2004).

L'utilizzo di luci con lunghezza d'onda limitate (es. verde, $450-620 \mathrm{~nm}$ ) possono risultare protettive in funzione però dell'atmosfera gassosa a ridosso dell'alimento e dovrebbero comunque riscontrare il favore del consumatore in fase di esposizione per la vendita. Durante il periodo di conservazione non si è osservato lo sviluppo di muffe 0 altri segni di alterazione se non la perdita di colore.

\section{Conclusioni}

Anche se non è stato oggetto del presente lavoro è importante ricordare che la luce, in presenza di sostanze fotosensibilizzanti, induce l'ossidazione anche delle proteine determinando la degradazione della metionina con formazione di metionale successivamente convertito in mercaptano, solfuri e disolfuri, responsabili dello sviluppo di off-flavor quale l'odore di pelle bruciata (derivante dalla fotossidazione della metionina), e l'odore di carta carbone (derivante dall'ossidazione dei lipidi), quest'ultimo riscontrato al termine del periodo sperimentale. I cambiamenti sensoriali indotti nei formaggi dai fenomeni ossidativi si manifestano generalmente dopo 1-4 giorni a seconda del prodotto, tuttavia la complessità della componente aromatica di questi alimenti e la mancanza di conoscenza da parte del consu- 
matore di aromi e sapori non propri (es. fungo, pelle bruciata, cavolo, ecc.) fa sì che molto spesso tali modificazioni non siano percepite.

Se lo sbianchimento o i cambiamenti aromatici non sono oggetto di attenzione è però fondamentale ricordare che i fenomeni ossidativi comportano importanti perdite nutrizionali a carico di acido ascorbico, riboflavina, vitamina $A$, -carotene, vitamina $B_{6}$, vitamina $B_{12}$, vitamina $D$, vitamina $K$, acido folico e tocoferolo, oltre a determinare la formazione di composti tossici (Mortensen et al., 2004). In assenza di indicazioni normative è necessario sensibilizzare gli operatori del settore nei confronti di questa problematica. È quindi opportuno valutare le caratteristiche delle fonti di illuminazione impiegate soprattutto durante l'esposizione presso i punti vendita, il materiale di confezionamento ricercando la migliore soluzione in termini di protezione dalla radiazione luminosa ai fini di una maggiore tutela del consumatore.

L'Asiago d'allevo D.0.P. ha dimostrato una certa suscettibilità alla fotossidazione che dovrebbe essere ulteriormente approfondita. Questa valutazione preliminare deve essere completata valutando la perdita delle vitamine liposolubili e la formazione di ossidi del colesterolo.

\section{Bibliografia}

AOAC, 2000. Official methods of analysis. 17th ed., Association of Official Analytical Chemists, Gaithersburg, MD, USA.

Balzan S, Elia CA, Segato S, Tenti S, Lignitto L, Piras N, Granata A, Novelli E, 2007. Fatty acids profile of cow's milk produced from alpine grazing. Proceedings of 5th Euro Fed Lipid Congress. Gothemburg, 2007 Sept. 16-19, Sweden.

Bianchi M, Battaglini LM, Mimosi A, Lussiana C, Prina A, Massimo V, 2002. Il formaggio Ossolano. Uno studio per la caratterizzazione del territorio, dei sistemi produttivi zootecnici, e dei formaggi. Aspetti zootecnici. In: Regione Piemonte, ed. Il formaggio Ossolano. Documento finale II Italia-Svizzera. Regione Piemonte ed., Torino, pp 197-214.

Bugaud C, Buchin S, Coulon JB, Hauwuy A, Dupont D, 2001. Influence of the nature of alpine pastures on plasmin activity, fatty acid and volatile compound composition of milk. Lait 81:401-14.

Cardoso DR, Olsen K, Skibsted LH, 2007. Mechanism of deactivation of triplet-excited riboflavin by ascorbate, carotenoids, and tocopherols in homogeneous and heterogeneous aqueous food model systems. J Agr Food Chem 55:6285-91.

Chozas MG, Sans RG, Romero IV, 1993. Comparison of various procedures employed in the TBA test of Spanish edible vegetable oils. Lipid/Fat 95:225-7.

Deger D, Ashoor SH, 1987. Light-induced changes in taste, appearance, odor, and riboflavin content of cheese. J Dairy Sci 70:1371-6.

Foote CS, 1991. Definition of type-I and tipe-II photosensitized oxidation. Photochem Photobiol 54:659.

Havemose MS, Weisbjerg MR, Bredie WLP, Nielsen JH, 2004. Influence of feeding different types of roughage on the oxidative stability of milk. Int Dairy J 14:563-70.

He YY, An JY, Jiang LJ, 1998. EPR and spectrophotometric studies on free radicals ( 0 2(center dot-), Cysa-HB center dot-) and singlet oxygen (0-1(2)) generated by irradiation of cysteamine substituted hypocrellin B. Int J Radiat Biol 74:647-54.

Intawiwat N, Dahla AV, Pettersen MK, Skaret J, Rukke EO, Wold JP, 2011. Effect of different wavelength of light on the formation of photo-0xidation in Gouda-like cheese. Int Dairy J 21:531-9.

ISMEA, 2011. Gli acquisti domestici nazionali di formaggi e latticini. Le tendenze recenti, il comportamento d'acquisto, le attese. Istituto di servizi per il mercato agricolo alimentare ed., Roma, Italia. Disponibile al sito: http://www.ismea.it/flex/cm/pages/ ServeBLOB.php/L/TT/IDPagina/6608

Jones ST, Aryana KJ, Losso JN, 2005. Storage stability of lutein during ripening of Cheddar Cheese. J Dairy Sci 88:1661-70.

Juric M, Bertelsen G, Mortensen G, Petersen MA, 2003. Light-induced colour and aroma changes in sliced, modified atmosphere packaged semi-hard cheeses. Int Dairy J 13:239-49.

Kristensen D, Hansen E, Arndal A, Appelgren Trinderup R, Skibsted LH, 2001. Influence of light and temperature on the colour and oxidative stability of processed cheese. Int
Dairy J 11:837-43.

Kristensen D, Orlien V, Mortensen G, Brockhoff P, Skibsted LH, 2000. Lightinduced oxidation in sliced Havarti cheese packaged in modified atmosphere. Int Dairy J 10: 95-103.

Mele M, Contarini G, Cercaci L, Serra A, Buccioni A, Povolo M, Conte G, Funaro A, Banni S, Lercker G, Secchiari P, 2011. Enrichment of Pecorino cheese with conjugated linoleic acid by feeding dairy ewes with extruded linseed: effect on fatty acid and triglycerides composition and on oxidative stability. Int Dairy J 21:365-72.

Møller A, Saxholt E, 1996. [Levnedsmiddeltabeller]. [Volume in danese]. 4a ed. Levnedsmiddelstyrelsen - National Food Agency) ed., Copenhagen, Danimarca.

Mortensen G, Bertelsen G, Mortensen BK, Stapelfeldt H, 2004. Light-induced changes in packaged cheeses: a review. Int Dairy J 14:85-102.

Mortensen G, Sørensen J, Stapelfeldt H, 2002. Light-Induced 0xidation in Semihard Cheeses. Evaluation of Methods Used To Determine Levels of Oxidation. J Agr Food Chem 50:4364-70.

Novelli E, Tenti S, Franco S, Soardo E, Barcarolo R, Balzan S, Tealdo E, Segato S, 2002. Applicazione di un metodo semplificato per estrarre la frazione lipidica al fine di determinare il profilo acidico in latte e derivati. Atti 6 Congresso Italiano di Scienza e Tecnologia degli Alimenti, Cernobbio (C0), Italia, 2002, Sett. 18-19.

PASW Statistics 18, 2009. Versione 18.0 per Windows, SPSS Inc., Chicago, USA.

Rocculi P, Romani S, Venir E, Dalla Rosa M, Mastrocola D, 2003. Aspetti tecnologici di prodotti a base di frutta trasformata "al minimo" (IV gamma). Rivista di Frutticoltura e di Ortofloricoltura 65:23-31.

Segato S, Balzan S, Elia CA, Lignitto L, Granata A, Magro L, Contiero B, Andrighetto I, Novelli E, 2007. Effect of period of milk production and ripening on quality traits of Asiago cheese. It J Anim Sci 6(Suppl.1): 469-71.

Trobetas A, Badeka A, Kontominas MG, 2008. Light-induced changes in grated Graviera hard cheese packaged under modified atmospheres. Int Dairy J 18:1133-9. 